\title{
LOGARITHMIC ABELIAN VARIETIES, III: LOGARITHMIC ELLIPTIC CURVES AND MODULAR CURVES
}

\author{
TAKESHI KAJIWARA, KAZUYA KATO, AND \\ CHIKARA NAKAYAMA
}

\section{To Professor Luc Illusie}

\begin{abstract}
We illustrate the theory of log abelian varieties and their moduli in the case of log elliptic curves.
\end{abstract}

\section{Introduction}

The purpose of this part III of our series of papers is to illustrate our theory of log abelian varieties in the case of 1-dimensional log abelian varieties, that is, log elliptic curves.

The theory of log abelian varieties is a new formulation of degenerating abelian varieties. A log abelian variety is something like a proper singular variety appearing when an abelian variety degenerates, but it still has a group structure. It is also like a smooth object in the sense of log geometry. Hence, we can develop the theory of log abelian varieties exactly parallel to the classical theory of nondegenerating abelian varieties. The purpose of this series of papers is to establish the theory of log abelian varieties of any dimension, and the aim of this Part III is to describe an essence of ideas by giving a complete proof for a typical case, that is, the 1-dimensional case. Thus, we hope that this paper gives a nice picture of degenerating elliptic

Received August 9, 2011. Revised March 23, 2012. Accepted May 10, 2012.

2010 Mathematics Subject Classification. Primary 14K10; Secondary 14J10, 14 D06.

Kajiwara's work was partially supported by Japan Society for the Promotion of Science (JSPS) Grant-in-Aid for Scientific Research (C)21540032.

Kato's work was partially supported by National Science Foundation grant DMS1001729.

Nakayama's work was partially supported by JSPS Grants-in-Aid for Scientific Research (C)18540017, (C)22540011. 
curves in the log world and thereby serves to introduce the readers to the whole theory of log abelian varieties.

Readers should notice that our emphasis always lies in the fact that the theory of log abelian varieties is parallel to the theory of abelian varieties; that is, the log geometric method can treat the degenerate objects as if they were not degenerate. For example, since a log elliptic curve $E$ has a group structure, the $\Gamma(N)$-level structure $(N \geqq 1)$ on it can be defined just as an isomorphism between $(\mathbb{Z} / N \mathbb{Z})^{2}$ and $\operatorname{Ker}(E \stackrel{N}{\rightarrow} E)$, and we can consider the moduli of $\log$ elliptic curves with $\Gamma(N)$-structure. These moduli are represented by nothing but the Deligne-Rapoport compactifications of modular curves (see [2]), so we can compare log elliptic curves and generalized elliptic curves in [2]. In fact, some of our proofs in this paper are based on the work of Deligne and Rapoport, but there are some important differences. For instance, a log elliptic curve can have $N$-level structures for various $N$, while the presence of an $N$-level structure determines the shape of a generalized elliptic curve. So, log elliptic curves can be useful when one studies a degenerating family of elliptic curves with level structures in changing levels. See Remark 5.2 for a more precise form of this remark.

The paper is organized as follows. In Section 1, we give a definition of log elliptic curves, which are nothing but 1-dimensional log abelian varieties, as defined in Part II [3] of our series. In Section 2, we define the moduli functors of log elliptic curves and review Deligne-Rapoport compactifications endowed with the natural log structures, which represent the moduli functors (see Theorem 5.1), as we prove in Section 5 after the preparations in Sections 3 and 4.

In Section 3, we construct universal families $\mathcal{E}^{\log }$ of $\log$ elliptic curves over Deligne-Rapoport compactifications (endowed with the natural log structures). These families induce by definition the morphism from the functor represented by Deligne-Rapoport compactifications on the category of log schemes to the moduli functor of log elliptic curves in Section 2, which is proved to be an isomorphism in Section 5 by standard techniques with models of log elliptic curves discussed in Section 4.

For simplicity, until Section 5, we limit ourselves to the most basic case in the sense that we do not use Drinfeld level structures, $\Gamma_{0}(N)$-level structures, and stacks, because the most basic case is enough for our purpose to show that, in the log world, the story of the moduli of degenerate objects exactly parallels that of the moduli of classical, nondegenerate objects. We 
plan to investigate these variants in forthcoming parts in this series of papers with full generality. For the reader's convenience, in Section 6 , we briefly discuss these generalizations, which also should be parallel to the nondegenerating stories, mentioning other works (e.g., Katz and Mazur [5] and Conrad [1]).

In the appendix, we give an alternative construction of the universal log elliptic curves $\mathcal{E}^{\log }$ in Section 3, which includes a precise relationship between our $\mathcal{E}^{\log }$ and the Deligne-Rapoport universal generalized log elliptic curves.

\section{§1. Log elliptic curves}

Let $S$ be an fs (fine and saturated) $\log$ scheme. Let (fs $/ S$ ) be the category of fs $\log$ schemes over $S$. Recall that a morphism $X \rightarrow Y$ in (fs/S) is strict if the $\log$ structure of $X$ is the pullback one from $Y$. We endow (fs $/ S$ ) with the strict étale topology (i.e., the topology by the strict morphisms whose underlying morphisms of schemes are étale in the usual sense).

In this section, we review the definition of log abelian varieties in $[3$, Definition 4.1] in the case where the dimension is 1 , that is, the case of log elliptic curves.

\section{1 .}

We begin with a typical example of log elliptic curves.

First, define two sheaves $\mathbb{G}_{m}$ and $\mathbb{G}_{m}$,log of abelian groups on (fs/ $S$ ) as follows. For an object $U$ of (fs $/ S)$,

$$
\begin{aligned}
\mathbb{G}_{m}(U) & =\Gamma\left(U, \mathcal{O}_{U}^{\times}\right), \\
\mathbb{G}_{m, \log }(U) & =\Gamma\left(U, M_{U}^{\mathrm{gp}}\right),
\end{aligned}
$$

where $M_{U}^{\mathrm{gp}}$ is the sheaf of abelian groups associated to $M_{U}$.

Next, let $q$ be a global section of $M_{S}$. Define a subgroup sheaf $\mathbb{G}_{m, \log }^{(q)}$ (see $\left[3\right.$, Section 1.3]) of $\mathbb{G}_{m, \log }$ as follows:

$$
\begin{aligned}
\mathbb{G}_{m, \log }^{(q)}(U)= & \left\{\varphi \in \Gamma\left(U, M_{U}^{\mathrm{gp}}\right) \mid \text { locally on } U,\right. \\
& \text { there exist } \left.i, j \in \mathbb{Z} \text { such that } q^{i}|\varphi| q^{j}\right\} .
\end{aligned}
$$

Here, by abuse of notation, we denote the image of $q$ in $M_{U}^{\mathrm{gp}}(U)$ by the same letter. For local sections $a, b \in M_{U}^{\mathrm{gp}}, a \mid b$ means that $a^{-1} b \in M_{U}$.

Let $q$ be a global section of $M_{S} / \mathcal{O}_{S}^{\times}$. Then, the subgroup sheaf $\mathbb{G}_{m, \log }^{(\tilde{q})}$ of $\mathbb{G}_{m, \log }$ is independent of a local lift $\tilde{q} \in M_{S}$ of $q$ and hence can be defined globally. We denote it again by $\mathbb{G}_{m, \log }^{(q)}$ by abuse of notation. 
EXAMPLE 1.2. Two typical examples of log elliptic curves are as follows.

(1) Let $E \rightarrow S$ be a strict morphism of fs log schemes. If the underlying morphism of schemes is an elliptic curve in the classical sense, $E$ is a $\log$ elliptic curve over $S$. We identify $E$ with the sheaf on (fs $/ S$ ) that it represents. We call log elliptic curves of this type elliptic curves.

(2) Let $q$ be a global section of $M_{S}$ as in Section 1.1. Assume that the image of $q$ in $\mathcal{O}_{S}$ is locally nilpotent. Then, the group sheaf $\mathbb{G}_{m, \log }^{(q)} / q^{\mathbb{Z}}$ on $(\mathrm{fs} / S)$ is a log elliptic curve. We call log elliptic curves of this type log Tate curves.

Now, a general log elliptic curve is a family consisting of the above two examples.

Definition 1.3. A log elliptic curve over an fs log scheme $S$ is a sheaf $E$ of abelian groups on (fs/S) satisfying the following three conditions.

(1) For any $s \in S$, the pullback of $E$ over $\bar{s}$ either is a log scheme strict over $\bar{s}$ whose underlying scheme is an elliptic curve or is isomorphic to a $\log$ Tate curve $\mathbb{G}_{m, \log }^{(q)} / q^{\mathbb{Z}}$ (Example 1.2). Here $\bar{s}$ is the Spec of the separable closure $\bar{k}$ of the residue field $k$ of $s$ endowed with the pullback $\log$ structure of $S$, and $q$ is a section of $M_{\bar{s}}$ whose image in $\bar{k}$ is zero.

(2) There exist a 1-dimensional semi-abelian scheme $G$ over $S$, a global section $q$ of $M_{S} / \mathcal{O}_{S}^{\times}$, and an exact sequence

$$
0 \longrightarrow G \longrightarrow E \longrightarrow \mathbb{G}_{m, \log }^{(q)} / \mathbb{G}_{m} q^{\mathbb{Z}} \longrightarrow 0 .
$$

(3) The diagonal morphism $E \rightarrow E \times E$ is represented by finite morphisms. (Equivalently, the 0 -section $S \rightarrow E$ is represented by finite morphisms.)

Note that a log elliptic curve is not necessarily represented by a log scheme; that is, it is an object of a different nature than a log scheme. On the other hand, we study its big representable subfunctors, called models, in Section 4.

Proposition 1.4. The above log elliptic curves are 1-dimensional log abelian varieties in the sense of [3, Definition 4.1]. Conversely, 1dimensional log abelian varieties in the sense of [3, Definition 4.1] are log elliptic curves.

Proof. First, in Example 1.2, (1) and (2) are log abelian varieties with constant degeneration in the sense of [3, Section 3]. Conversely, let $A$ be a 
log abelian variety with constant degeneration. If its semi-abelian part $G$ is 1-dimensional, it is an elliptic curve or a torus. Then, by [3, Theorem 3.4], $A$ is either a strict elliptic curve or a log Tate curve (Example 1.2), respectively. Hence, the first condition in Definition 1.3 and that in [3, Definition 4.1] are equivalent.

Next, it is easy to see that a log elliptic curve over $S$ satisfies the condition $\left[3\right.$, second condition of 4.1.2] if we take $X=Y=\mathbb{Z}$ and $\langle m, n\rangle:=q^{m n}$ and if $\phi$ is the identity.

The third condition in Definition 1.3 is identical to that in $[3$, Definition 4.1].

It remains to show that a $\log$ abelian variety $A$ satisfies Definition $1.3(2)$. By [3, Section 4.4], $G$ exists globally. We show that $A / G$ is isomorphic to $\mathbb{G}_{m, \log }^{(q)} / \mathbb{G}_{m} q^{\mathbb{Z}}$ for some $q$. To see this, we can work locally on $S$ because $q$ is uniquely determined by $E$ if it exists (see [3, Theorem 7.6(2)]). Consider the locus $Z \subset S$ where $G$ is a torus. Then, $A$ is with constant degeneration on $Z$ by [3, Theorem 4.6(1)]. Hence, by the argument of the first paragraph of this proof, $\left.A\right|_{Z}$ is isomorphic to a log Tate curve $\mathbb{G}_{m, \log }^{(q)} / q^{\mathbb{Z}}$ (Example 1.2). Hence, $A / G=\mathbb{G}_{m, \log }^{(q)} / \mathbb{G}_{m} q^{\mathbb{Z}}$ on $Z$. On the other hand, we have $A / G=$ $\mathcal{H}$ om $\left(X, \mathbb{G}_{m, \log } / \mathbb{G}_{m}\right)^{(Y)} / \bar{Y}$ on $S$ for an admissible pairing $\langle\rangle:, X \times Y \rightarrow$ $\mathbb{G}_{m, \log } / \mathbb{G}_{m}$. Take a local extension of $q$ over $S$, still denoted by $q$. It is enough to show that $q$ is invertible outside $Z$. But, by [3, Theorem 7.6(2)], we may assume that $q$ is the image of some element of $X \times Y$ in $M_{S} / \mathcal{O}_{S}^{\times}$which vanishes outside $Z$. Hence, $q$ is invertible outside $Z$. Thus, Definition 1.3(2) is satisfied.

We note that, as seen in the above proof, $G$ and $q$ in Definition 1.3(2) are uniquely determined by $E$.

Finally in this section, we define some standard level structures on a log elliptic curve. By virtue of the remarkable fact that a log elliptic curve is a commutative group object, the definitions are parallel to the classical ones.

Definition 1.5. Let $N \geq 1$. Let $S$ be an fs log scheme over $\operatorname{Spec} \mathbb{Z}[1 / N]$.

(1) A $\Gamma(N)$-structure of a log elliptic curve $E$ over $S$ is an isomorphism $(\mathbb{Z} / N \mathbb{Z})^{2} \rightarrow \operatorname{Ker}(E \stackrel{N \text {-times }}{\longrightarrow} E)$.

(2) A $\Gamma_{1}(N)$-structure of a log elliptic curve $E$ over $S$ is a section $P: S \rightarrow E$ of exact order $N$, that is, of order $N$ in each geometric fiber.

(3) $\mathrm{A} \Gamma_{0}(N)$-structure of a log elliptic curve $E$ over $S$ is a subgroup scheme $C$ of $\operatorname{Ker}(E \stackrel{N \text {-times }}{\longrightarrow} E)$ that is étale locally isomorphic to $\mathbb{Z} / N \mathbb{Z}$. 
REMARK 1.6. For any log elliptic curve over $S$, the above structures exist locally on $S$ in the Kummer-étale topology — which we abbreviate as the két topology; that is, there is a surjective, log étale morphism $S^{\prime} \rightarrow S$ of Kummer type (see [6]) such that the pullback log elliptic curve over $S^{\prime}$ has the above structures.

\section{$\S 2$. Moduli functors}

Let $N \geq 1$. We define moduli functors of log elliptic curves with $\Gamma_{*}(N)$ structure, where $*=\emptyset, 1$. We will explain the case of $\Gamma_{0}(N)$ in Section 6 .

Definition 2.1. Let $S$ be an fs log scheme over Spec $\mathbb{Z}[1 / N]$. We define four functors $F_{N}, \bar{F}_{N}, F_{N, 1}$, and $\bar{F}_{N, 1}:($ fs $/ S) \rightarrow$ (set) as follows. Let $U$ be an object of (fs $/ S)$.

(1) Let $F_{N}(U)$ be the set of isomorphism classes of elliptic curves $E$ over $U$ with $\Gamma(N)$-structure.

(2) Let $\bar{F}_{N}(U)$ be the set of isomorphism classes of log elliptic curves $E$ over $U$ with $\Gamma(N)$-structure.

(3) Let $F_{N, 1}(U)$ be the set of isomorphism classes of elliptic curves $E$ over $U$ with $\Gamma_{1}(N)$-structure.

(4) Let $\bar{F}_{N, 1}(U)$ be the set of isomorphism classes of log elliptic curves $E$ over $U$ with $\Gamma_{1}(N)$-structure.

Here an elliptic curve $E$ over $U$ is an fs log scheme $E$ over $U$ such that $E \rightarrow U$ is a strict morphism whose underlying morphism is an elliptic curve in the usual sense (Example 1.2(1)). Since we always regard such an $E$ as a log elliptic curve, we have

$$
F_{N} \subset \bar{F}_{N}, \quad F_{N, 1} \subset \bar{F}_{N, 1}
$$

\section{2 .}

Next, we recall the Deligne-Rapoport compactifications of moduli of elliptic curves with level structure [2].

We work over $\mathbb{Z}[1 / N]$.

First, let $N \geq 3$. Consider the functor $(\operatorname{sch} / \mathbb{Z}[1 / N]) \rightarrow$ (set) associating to a scheme $U$ over $\mathbb{Z}[1 / N]$ the set of isomorphism classes of elliptic curves over $U$ with $\Gamma(N)$-structures. Then, this functor is represented by a scheme, which is denoted by $Y(N)$.

Let $N \geq 4$. The functor $(\operatorname{sch} / \mathbb{Z}[1 / N]) \rightarrow($ set) associating to $U$ the set of isomorphism classes of elliptic curves over $U$ with $\Gamma_{1}(N)$-structures is also represented by a scheme, which is denoted by $Y_{1}(N)$. 
Next, let $N \geq 3$ (resp., $N \geq 4$ ). The functor $(\operatorname{sch} / \mathbb{Z}[1 / N]) \rightarrow$ (set) associating to $U$ the set of isomorphism classes of generalized elliptic curves over $U$ with $\Gamma(N)$-structures (resp., $\Gamma_{1}(N)$-structures) is represented by a scheme, which is denoted by $X(N)$ (resp., $X_{1}(N)$ ).

Then, both $X(N)$ and $X_{1}(N)$ are proper and smooth over $\mathbb{Z}[1 / N]$; the scheme $Y(N)$ (resp., $\left.Y_{1}(N)\right)$ is an open subscheme of $X(N)$ (resp., $X_{1}(N)$ ) whose complement is a relative normal crossing divisor over $\mathbb{Z}[1 / N]$. We call the components of this divisor cusps.

We endow $X(N)$ (resp., $X_{1}(N)$ ) with the fs $\log$ structure defined by the cusps, that is, by the divisor $X(N)-Y(N)$ (resp., $X_{1}(N)-Y_{1}(N)$ ).

Let $\mathcal{E}$ be the universal generalized elliptic curve over $X(N)$. Endow $\mathcal{E}$ with the fs $\log$ structure defined by the divisor which is the pullback of $X(N)-Y(N)$.

Let $S$ be an fs log scheme over $\mathbb{Z}[1 / N]$. In the following, we denote the base change of $Y(N), Y_{1}(N), X(N), X_{1}(N)$ to $S$ by the same symbol. By definition, the functor $F_{N}$ (resp., $F_{N, 1}$ ) in Definition 2.1 is represented by the fs $\log$ scheme $Y(N)$ (resp., $\left.Y_{1}(N)\right)$.

In Section 5, we will prove that moduli functors in Definition 2.1 are represented by Deligne-Rapoport compactifications in Section 2.2, after the preparations in Sections 3 and 4.

\section{$\S 3$. Universal log elliptic curves}

In this section, we construct universal log elliptic curves over $X(N)$ over $\mathbb{Z}[1 / N]$. Fix $N \geq 3$ in this section.

Let $\mathcal{G}$ be the universal semi-abelian scheme over $X(N)$, that is, the largest semi-abelian subscheme of the universal generalized elliptic curve $\mathcal{E}$ over $X(N)$ (see Section 2.2). Then, $\mathcal{G}$ coincides with the open subspace of $\mathcal{E}$ where the structure map $\mathcal{E} \rightarrow X(N)$ is strict.

Below, we will compactify $\mathcal{G}$ around each cusp as we have done in [3, Proposition 4.8] to get our universal log elliptic curve. Thus, we do not use $\mathcal{E}$ itself.

See the appendix for an alternative construction of the universal log elliptic curves, which uses $\mathcal{E}$ and shows the precise relationship between our construction and $\mathcal{E}$.

\section{1 .}

Let $q$ be the local parameter of the cusp of the moduli stack $X(1)$ of elliptic curves. Let $J$ be the set of cusps of $X(N)$. For each $v \in J$, let $O_{v}$ be 
the completion of the local ring of $X(N)$ at $v$, and let $K_{v}$ be its fraction field. Then, we have $O_{v}=\mathbb{Z}\left[1 / N, \zeta_{N}\right]\left[\left[q^{1 / N}\right]\right]$ and $K_{v}=\mathbb{Z}\left[1 / N, \zeta_{N}\right]\left[\left[q^{1 / N}\right]\right][1 / q]$, where $\zeta_{N}$ is a primitive $N$ th root of unity. Endow $\operatorname{Spec} O_{v}$ and $\operatorname{Spec} K_{v}$ with the inverse image log structures of $X(N)$, which are the log structure defined by $q^{1 / N}$ and the trivial log structure, respectively.

\subsection{The $q_{v}$-Tate curve}

Let $q_{v}=q$, and let $\mathcal{E}_{q_{v}}$ be the $q_{v}$-Tate curve, which we will review briefly (see [3, Section 1] for details). For each integer $i \geq 1$ and a nonempty subset $I$ of $\mathbb{Q}$ stable under the translation by $\mathbb{Z}$ such that $I / \mathbb{Z}$ is finite, we construct the proper fs $\log$ scheme $\mathcal{E}_{q_{v}^{i}}^{(I)}$ over $\operatorname{Spec} O_{v}$ by the log Mumford construction (see $\left[3\right.$, Section 1]). For example, $\mathcal{E}_{q_{v}}^{((1 / N) \mathbb{Z})}$ is nothing but the base change of $\mathcal{E}$ to $\operatorname{Spec} O_{v}$ (see [3, Section 1.8]). Over $v$, the pullback of $\mathcal{E}_{q_{v}^{i}}^{(I)}$ is $\mathbb{G}_{m, \log }^{\left(q_{v}^{i}, I\right)} / q_{v}^{i \mathbb{Z}}$, where $\mathbb{G}_{m, \log }^{\left(q_{v}^{i}, I\right)}$ is the subsheaf of $\mathbb{G}_{m, \log }$ consisting of sections locally satisfying $q^{\alpha}|x| q^{\alpha^{\prime}}$ for some $\alpha \in I$. Here $\alpha^{\prime} \in I$ is the smallest element of $\{\beta \in I \mid \beta>\alpha\}$, and $q^{\alpha}|x| q^{\alpha^{\prime}}$ means that $q^{\alpha n}\left|x^{n}\right| q^{\alpha^{\prime} n}$ for some positive integer $n$ with $\alpha n$ and $\alpha^{\prime} n$ integers. Then $\mathcal{E}_{q_{v}}=\left(\bigsqcup_{(i, I)} \mathcal{E}_{q_{v}^{i}}^{(I)}\right) / \sim$, where $\sim$ is the equivalence relation in the category of sheaves on (fs/Spec $\left.O_{v}\right)$ generated by the following two equivalences (see [3, Section 1.6]).

(1) For any $(i, I)$ and $(j, J)$ such that $j \mid i$ and such that $(i / j) I \supset J$, any element of $\mathcal{E}_{q_{v}^{i}}^{(I)}(U)$ for any fs log scheme $U$ over $\operatorname{Spec} O_{v}$ is equivalent to its image in $\mathcal{E}_{q_{v}^{j}}^{(J)}(U)$ under the canonical morphism $\mathcal{E}_{q_{v}^{i}}^{(I)} \rightarrow \mathcal{E}_{q_{v}^{j}}^{(J)}$.

(2) For any $(i, I)$, if we denote $J=I+(1 / i)$, any element of $\mathcal{E}_{q_{v}^{i}}^{(I)}(U)$ for any fs $\log$ scheme $U$ over $\operatorname{Spec} O_{v}$ is equivalent to its image in $\mathcal{E}_{q_{v}^{i}}^{(J)}(U)$ under the morphism $\mathcal{E}_{q_{v}^{i}}^{(I)} \rightarrow \mathcal{E}_{q_{v}^{i}}^{(J)} ; \varphi \mapsto q_{v} \varphi$.

\section{3 .}

We define a log elliptic curve $\mathcal{E}^{\log } \rightarrow X(N)$ as the sheaf that makes the diagram

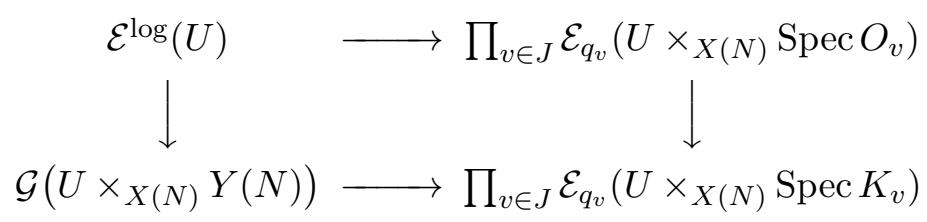

Cartesian for each $X(N)$-fs log scheme $U$, similar to the ones in [3, Proposition 4.7]. 
We show that this $\mathcal{E}^{\log }$ is actually a log elliptic curve (Definition 1.3).

Proposition 3.4. The above construction gives a log elliptic curve.

We call this $\mathcal{E}^{\log }$ a universal log elliptic curve.

Proof. We can prove this exactly in a way parallel to [3, Proposition 4.8] as follows.

We have to check the three conditions in Definition 1.3. In the same way as in [3, Section 9.10], we can show that there is an exact sequence

$$
0 \rightarrow \mathcal{G} \rightarrow \mathcal{E}^{\log } \rightarrow \bigoplus_{v \in J} i_{v *}\left(\mathbb{G}_{m, \log }^{\left(q_{v}\right)} /\left(\mathbb{G}_{m} \cdot q_{v}^{\mathbb{Z}}\right)\right) \rightarrow 0
$$

where $i_{v}$ is the inclusion morphism $v \rightarrow X(N)$. This implies Definition 1.3(2). Next, again as in [3, Section 9.10], we can prove that the pullback of $\mathcal{E}^{\log }$ to the open subset $Y(N)$ of $X(N)$ coincides with $\mathcal{G}$ and that the pullback $\left.\mathcal{E}^{\log }\right|_{O_{v}}$ of $\mathcal{E}^{\log }$ to $\operatorname{Spec}\left(O_{v}\right)$ is $\mathcal{E}_{q_{v}}$ for each $v \in J$. This shows Definition 1.3(1). Finally, Definition 1.3(3) also can be checked similarly as in [3, Section 9.10]. So, we have finished.

The universal log elliptic curve has the canonical level structure $(\mathbb{Z} /$ $N \mathbb{Z})^{2} \rightarrow \mathcal{E}^{\log }$ coming from the level structure of $\left.\mathcal{G}\right|_{Y(N)}$ and those of the $q_{v}$-Tate curves.

\section{§4. Models}

In this section, we consider representable models of log elliptic curves, which are used in the proof of Theorem 5.1 in Section 5.

\subsection{The sheaf $\mathcal{C}^{\Sigma}$}

Let $S$ be an fs $\log$ scheme. Let $\mathcal{C}:=\mathbb{G}_{m, \log } / \mathbb{G}_{m}$ on $(\mathrm{fs} / S)$. Let $q$ be a global section of $M_{S} / \mathcal{O}_{S}^{\times}$. For a bounded rational interval $I:=\left[\alpha, \alpha^{\prime}\right]$ of $\mathbb{Q}$, $\alpha, \alpha^{\prime} \in \mathbb{Q}$, and $\alpha \leq \alpha^{\prime}$, we define a sheaf $\mathcal{C}^{I}$ as the subsheaf of $\mathcal{C}$ consisting of sections $x$ locally satisfying $q^{\alpha}|x| q^{\alpha^{\prime}}$. Here $q^{\alpha}|x| q^{\alpha^{\prime}}$ means that $q^{\alpha n}\left|x^{n}\right| q^{\alpha^{\prime} n}$ for some positive integer $n$ with $\alpha n$ and $\alpha^{\prime} n$ integers. For a collection $\Sigma=$ $\left(I_{i}\right)_{i}$ of bounded rational intervals such that for any $i, j$, the intervals $I_{i}$ and $I_{j}$ have no common interior point, let $\mathcal{C}^{\Sigma}=\bigcup_{i} \mathcal{C}^{I_{i}}$.

\subsection{The sheaf $\mathcal{Q}^{\Sigma}$}

Further, let $E$ be a log elliptic curve over $S$. Let

$$
0 \longrightarrow G \longrightarrow E \longrightarrow \mathcal{Q} \longrightarrow 0
$$


be the exact sequence as in Definition 1.3, where $\mathcal{Q}:=\mathbb{G}_{m, \log }^{(q)} / \mathbb{G}_{m} q^{\mathbb{Z}}$. We assume that $\Sigma$ is preserved by the translation $\alpha \mapsto \alpha+1$. Let $\mathcal{Q}^{\Sigma}$ be the image of the natural projection $\mathcal{C}^{\Sigma} \rightarrow \mathcal{Q}$. Let $E^{(\Sigma)} \subset E$ be the pullback of $\mathcal{Q}^{\Sigma}$ by $E \rightarrow \mathcal{Q}$. Then $E^{(\Sigma)}$ is represented by an fs log scheme which is log smooth over $S$ [3, Section 1.5]. When $\bigcup I_{i}=\mathbb{Q}$, this fs log scheme is also proper over $S$.

\subsection{Representable models of log elliptic curves}

Let the assumption be as in Section 4.1. In the rest of Section 4, fix a $\Sigma$ which is preserved by $\alpha \mapsto \alpha+1$. Assume that $\bigcup I_{i}=\mathbb{Q}$. Let $G$ be a 1 dimensional semi-abelian scheme over $S$. Let $W$ be a sheaf of sets on (fs $/ S$ ) endowed with a diagram

$$
G \stackrel{i}{\rightarrow} W \stackrel{p}{\rightarrow} \mathcal{Q}^{\Sigma}
$$

with $i$ injective and $p$ surjective such that $p \circ i$ is the trivial map.

Let $(W \times W)^{\prime}$ be the subsheaf of $W \times W$ defined by $\{(x, y) \mid p(x) p(y) \in$ $\mathcal{Q}$ belongs to $\mathcal{Q}^{\Sigma}$. A map

$$
(W \times W)^{\prime} \rightarrow W
$$

is called a partial group law on such a $W$ if the following three conditions (a)-(c) are satisfied. We say that $x y$ is defined if $(x, y) \in W \times W$ belongs to $(W \times W)^{\prime}$.

(a) For any $x, y, z \in W$, if $x y,(x y) z, y z$ are defined, then $(x y) z=x(y z)$. (Note that the right-hand side is defined by the assumptions.)

(b) For any $x \in W, 1_{G} x=x$. Here $1_{G}$ is the unit section of $G$.

(c) For any $x, y \in W$, if $x y$ is defined, then $x y=y x$. (Note that $y x$ is also defined if $x y$ is defined.)

Since $(G \times W) \cup(W \times G) \subset(W \times W)^{\prime}$, a partial group law induces an action of $G$ on $W$.

We consider three more conditions.

(d) The morphism $i$ is compatible with the (partial) group laws of $G$ and $W$.

(e) The morphism $p$ is compatible with the partial group laws of $W$ and $\mathcal{Q}^{\Sigma}$, where the partial group law on $\mathcal{Q}^{\Sigma}$ is the natural one.

(f) The sheaf $W$ is a $G$-torsor over $\mathcal{Q}^{\Sigma}$; that is, $G \times{ }_{S} W \rightarrow W \times{ }_{\mathcal{Q}^{\Sigma}} W$; $(g, w) \mapsto(g w, w)$ is bijective.

Let $\mathcal{W}$ be the category of $(W, i, p)$ as above endowed with a partial group law satisfying (d)-(f). The morphisms of this category are defined naturally. 
It is easy to see that the correspondence $E \mapsto E^{(\Sigma)}:=$ the pullback of $\mathcal{Q}^{\Sigma}$ by $E \rightarrow \mathcal{Q}$ defines a functor $\mu$ from $\operatorname{Ext}(\mathcal{Q}, G)$ to $\mathcal{W}$.

Proposition 4.4. Let the notation be as in the previous paragraph. Assume that there is an interval $I$ in $\Sigma$ that contains $0 \in \mathbb{Q}$ in its interior.

Let $\mathcal{A}$ be the category of log elliptic curves $E$ over $S$ satisfying the following conditions.

(i) The semi-abelian part of $E$ is $G$.

(ii) The log étale quotient $E / G$ is $\mathcal{Q}$.

Let $\mathcal{B}$ be the full subcategory of $\mathcal{W}$ consisting of objects satisfying the following conditions.

(g) For any $s \in S$, either the pullback of $G \rightarrow W$ to $\bar{s}$ is an isomorphism of elliptic curves and $q$ is invertible, or the pullback of $G \rightarrow W \rightarrow \mathcal{Q}^{\Sigma}$ to $\bar{s}$ is isomorphic to $\mathbb{G}_{m} \rightarrow \mathbb{G}_{m, \log }^{(\Sigma)} / \tilde{q}^{\mathbb{Z}} \rightarrow \mathcal{Q}^{\Sigma}$ and $q$ is not invertible, where $\tilde{q}$ is a lift of $q$.

(h) The sheaf $W$ is quasi-separated over $S$.

Then the functor $\mu$ in the above induces an equivalence of the categories $\mathcal{A}$ and $\mathcal{B} ; E \mapsto E^{(\Sigma)}$.

Proof. First we prove that $\mu: \operatorname{Ext}(\mathcal{Q}, G) \rightarrow \mathcal{W}$ is an equivalence by giving the inverse functor. Let $W$ be an object of $\mathcal{W}$. Let $I$ be the interval in the assumption. Let $\widetilde{W^{I}}:=W \times{ }_{\mathcal{Q}^{\Sigma}} \mathcal{C}^{I}$. Let $\left(\widetilde{W}^{I} \times \widetilde{W}^{I}\right)^{\prime} \rightarrow \widetilde{W}^{I}$ be the induced partial group law, where $\left(\widetilde{W}^{I} \times \widetilde{W}^{I}\right)^{\prime}$ is the subsheaf of $\widetilde{W}^{I} \times \widetilde{W}^{I}$ defined by $\left\{(x, y) \mid\right.$ the product of the images of $x$ and $y$ in $\mathcal{Q}$ belongs to $\left.\mathcal{Q}^{I}\right\}$. Let $\widetilde{E}$ be the két sheafification of the presheaf (see [6] for the definition of the két topology; see Remark 1.6 in this paper) that associates to each $U \in(\mathrm{fs} / S)$ the abelian group defined by $\widetilde{W^{I}}(U)$ as a set of generators and $[x][y]=[x y]$ for all $(x, y) \in\left(\widetilde{W^{I}} \times \widetilde{W}^{I}\right)^{\prime}(U)$ as relations, where $[x]$ denotes the generator corresponding to $x \in \widetilde{W}^{I}(U)$.

Take an $n \geq 1$ such that $1 / n \in I$. Let $E$ be the quotient sheaf of $\widetilde{E}$ by the subgroup sheaf generated by a local section $\left[\left(w, q^{1 / n}\right)\right]^{n}$, where $w$ is the section of $W[n]$ whose image in $\mathcal{Q}^{\Sigma}$ is the image of $q^{1 / n}$, and $\left(w, q^{1 / n}\right)$ is regarded as a section of the fiber product $W \times{ }_{\mathcal{Q}^{\Sigma}} \mathcal{C}^{I}$. Then, it is straightforward to see that the map $W \mapsto E$ is well defined and gives an inverse of $\mu$.

Further, it is easy to see that $E \in \operatorname{Ext}(\mathcal{Q}, G)$ satisfies Definition 1.3(1) if and only if the corresponding $W$ satisfies condition $(\mathrm{g})$. It is also easy 
to see that if $E \in \operatorname{Ext}(\mathcal{Q}, G)$ is a log elliptic curve, then the corresponding $W$ satisfies condition (h). It remains to show its converse. Assume that the corresponding $W$ is quasi-separated. Then, $\widetilde{W^{I}}$ is quasi-separated. This implies that $\widetilde{E}^{(n I)}:=E \times{ }_{\mathcal{Q}} \mathcal{C}^{n I}$ is quasi-separated for all $n>0$. Then, we can see that the morphism $\widetilde{E}^{(n I)} \times_{E} \widetilde{E}^{(n I)} \rightarrow \widetilde{E}^{(n I)} \times_{S} \widetilde{E}^{(n I)}$ is quasi-compact. Since any morphism $U \rightarrow E \times{ }_{S} E$ from a quasi-compact fs log scheme locally factors through $\widetilde{E}^{(n I)} \times{ }_{S} \widetilde{E}^{(n I)}$ for some $n$, we conclude that $E$ is quasiseparated.

\section{$\S 5$. Representability}

In this section, we prove the following theorem on the representability of the moduli functors of log elliptic curves with level structure.

TheOREM 5.1. Let $N \geq 3$ (resp., $N \geq 4$ ), and let $S$ be an fs log scheme over Spec $\mathbb{Z}[1 / N]$ with the trivial log structure. Then the moduli functor $\bar{F}_{N}$ (resp., $\bar{F}_{N, 1}$ ) is represented by the Deligne-Rapoport compactification $X(N)$ (resp., $X_{1}(N)$ ) over $S$ with the log structure defined by the cusps.

REMARK 5.2. Let $K$ be a complete discrete valuation field, let $q$ be a nonzero element of the maximal ideal of the valuation ring $O_{K}$, and let $E_{q}$ be the Tate elliptic curve over $K$ with " $q$-invariant" $q$. We can ask if there is a best model among all proper models of $E_{q}$ over $O_{K}$. Since $X(N)$ is a fine moduli in the sense of Deligne and Rapoport and in the sense of the above theorem, by giving an $N$-level structure on $E_{q}$, we get two kinds of good models of $E_{q}$ over $O_{K}$, respectively. In the sense of Deligne and Rapoport, this good model is a generalized elliptic curve, which depends on $N$. On the other hand, in the sense of the above theorem, this good model is a log elliptic curve and independent of $N$. (See [3, Section 1.8] for more explanations.)

We prove 5.1. We prove the case of $X(N)$. The other case of $X_{1}(N)$ is similar and is omitted.

\subsection{Two properties of $\bar{F}_{N}$}

Using the universal log elliptic curve in Section 3, we have a morphism $i: P \rightarrow F$, where $P$ denotes the functor represented by $X(N)$ with log structures along cusps, and $F$ is the moduli functor $\bar{F}_{N}$ of log elliptic curves with level $N$-structure. We remark that both $P$ and $F$ are sheaves with respect to the étale topology. 
To show that $i$ is an isomorphism, it is enough to verify the following two statements.

(1) Finiteness: $F$ is locally of finite presentation.

(2) Uniqueness: For a complete Noetherian local ring $R$, the canonical morphism $F(R) \rightarrow$ proj $\lim _{n} F\left(R / m_{R}^{n}\right)$ is injective.

Here, $\operatorname{Spec} R$ is endowed with an fs $\log$ structure, and $\operatorname{Spec} R / m_{R}^{n}$ is endowed with the pullback log structure.

\subsection{Reduction to the two properties of $\bar{F}_{N}$}

We first explain that Theorem 5.1 follows from statements (1) and (2) above. Using (1), we have only to show that, for the strict Henselization $R$ endowed with an fs log structure of a local ring of a scheme of finite type over $\mathbb{Z}$, the morphism $P(R) \rightarrow F(R)$ is bijective. Moreover, by the Artin approximation theorem, we may assume that $R$ is complete.

For an integer $n \geq 0$, the morphism $P\left(R_{n}\right) \rightarrow F\left(R_{n}\right)$, where $R_{n}:=$ $R / m_{R}^{n+1}$, is bijective by using the equivalence between the category of log abelian varieties with constant degenerations and that of log 1-motives (see $[3$, Theorem 3.4]). Then statement (2) and a commutative diagram

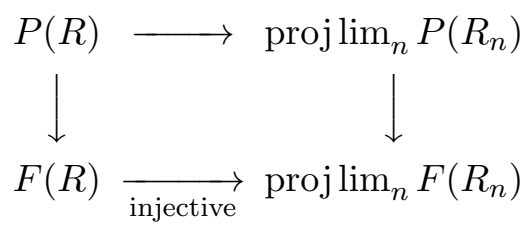

show that $i$ is an isomorphism because the upper horizontal arrow is an isomorphism.

\subsection{Beginning of the proof of finiteness}

We prove statement $(1)$. Let $\left(S_{i}\right)_{i}$ be a filtered projective system of affine fs $\log$ schemes whose transition morphisms are strict. Let $S:=\lim S_{i}$. Let $\left(E_{1}, \mu_{1}\right),\left(E_{2}, \mu_{2}\right) \in F\left(S_{i_{0}}\right)$ for some $i_{0}$, where $E_{j}$ is a log elliptic curve and $\mu_{j}$ is a level structure on it $(j=1,2)$. Assume that $\left.\left.\left(E_{1}, \mu_{1}\right)\right|_{S} \cong\left(E_{2}, \mu_{2}\right)\right|_{S}$. We will show that $\left.\left.\left(E_{1}, \mu_{1}\right)\right|_{S_{i}} \cong\left(E_{2}, \mu_{2}\right)\right|_{S_{i}}$ for some $i$. Consider the exact sequences in Definition 1.3(2) for $E_{j}(j=1,2)$ :

$$
0 \rightarrow G_{j} \rightarrow E_{j} \rightarrow \mathbb{G}_{m, \log }^{\left(q_{j}\right)} / \mathbb{G}_{m} q_{j}^{\mathbb{Z}} \rightarrow 0
$$

Since $\left.\left.G_{1}\right|_{S} \cong G_{2}\right|_{S}$ and $\left.q_{1}\right|_{S}=\left.q_{2}\right|_{S}$, we have $\left.\left.G_{1}\right|_{S_{i}} \cong G_{2}\right|_{S_{i}}$ and $\left.q_{1}\right|_{S_{i}}=\left.q_{2}\right|_{S_{i}}$ for some $i$. Hence, we may assume that $G_{1}=G_{2}=: G$ and that $q_{1}=q_{2}=: q$. 


\subsection{Use of a model}

Take $\Sigma=\{[n-(1 / 2), n+(1 / 2)] \mid n \in \mathbb{Z}\}$, and let $W_{j}=E_{j}^{(\Sigma)}(j=1,2)$. Since $\left.\left.W_{1}\right|_{S} \cong W_{2}\right|_{S}$, we have $\iota:\left.\left.W_{1}\right|_{S_{i}} \cong W_{2}\right|_{S_{i}}$ for some $i$. Enlarging $i$, we may assume that the isomorphism $\iota$ is compatible with the morphisms from $G$. Further, $\mathcal{Q}$ is locally of finite presentation. This is reduced to the fact that $\mathbb{G}_{m, \log } / \mathbb{G}_{m}$ is locally of finite presentation, which is easily checked. Hence, we may assume that $\iota$ is compatible with the morphisms to $\mathcal{Q}$. Furthermore, since $\left(W_{1} \times W_{1}\right)^{\prime}$ (in the notation of Section 4.3) is represented by a log blowup of $W_{1} \times_{S} W_{1}$, enlarging $i$, we may assume that $\iota$ is compatible with the partial group laws on $W_{1}$ and $W_{2}$. By Proposition 4.4, $\left.\left.E_{1}\right|_{S_{i}} \cong E_{2}\right|_{S_{i}}$. Hence, $\left.\left.E_{1}\right|_{S_{i}}[n] \cong E_{2}\right|_{S_{i}}[n]$. Lastly, enlarging $i$, we have $\left.\left.\mu_{1}\right|_{S_{i}} \cong \mu_{2}\right|_{S_{i}}$.

\subsection{Descent}

Next let $(E, \mu) \in F(S)$. We will prove that $(E, \mu)$ comes from an element of $F\left(S_{i}\right)$ for some $i$, which completes the proof of statement (1). We have an exact sequence

$$
0 \rightarrow G \rightarrow E \rightarrow \mathcal{Q} \rightarrow 0
$$

over $S$. First, $G$ and $q$ come from some $i$. Also, $W:=E^{(\Sigma)}$ is the base change of a proper fs log scheme $W$ (abuse of notation) over $S_{i}$ for some $i$. The morphism $G \rightarrow W$ also comes from some $i$. We may assume that it is strict and that it is a monomorphism. Since $\mathcal{Q}^{\Sigma}$ is locally of finite presentation, the morphism $W \rightarrow \mathcal{Q}^{\Sigma}$ also comes from some $i$. Again, by the fact that $\mathcal{Q}^{\Sigma}$ is locally of finite presentation, we may assume that the composite $G \rightarrow W \rightarrow \mathcal{Q}^{\Sigma}$ is trivial.

\subsection{The condition (g)}

Enlarging $i$ further, we may assume the equality of two subsets $U_{i}:=\{s \in$ $S_{i} \mid$ the pullback of $G$ to $s$ is an elliptic curve $\}=\left\{s \in S_{i} \mid q\right.$ is invertible at $s\}$ of $S_{i}$ because the pullbacks of the two subsets to $S$ coincide. Enlarging $i$, we may assume that $G \rightarrow W$ is an isomorphism on $U_{i}$. Let $Z_{i}=S_{i}-U_{i}$, and let $Z=\lim Z_{i}$. By [3, Theorem 4.6(1)], the pullback of $E$ to $Z$ is a $\log$ abelian variety with constant degeneration. Hence, $G \rightarrow W \rightarrow \mathcal{Q}^{\Sigma}$ on $Z$ is isomorphic to $\mathbb{G}_{m} \rightarrow \mathbb{G}_{m, \log }^{(\Sigma)} / \tilde{q}^{\mathbb{Z}} \rightarrow \mathcal{Q}^{\Sigma}$. Therefore, enlarging $i$, we may assume that $G \rightarrow W \rightarrow \mathcal{Q}^{\Sigma}$ on $Z_{i}$ is isomorphic to $\mathbb{G}_{m} \rightarrow \mathbb{G}_{m, \log }^{(\Sigma)} / \tilde{q}^{\mathbb{Z}} \rightarrow \mathcal{Q}^{\Sigma}$. Thus, we may assume that condition (g) in Proposition 4.4 is satisfied. 


\subsection{Surjectivity of $W \rightarrow \mathcal{Q}^{\Sigma}$}

Further, we may assume that condition (h) in Proposition 4.4 is also satisfied. Then, we can prove that $W \rightarrow \mathcal{Q}^{\Sigma}$ is surjective as follows. To see this, we may assume that $S$ is of finite type over $\mathbb{Z}$. Let $T$ be an fs $\log$ scheme over $S$. We have to prove that a section of $\mathcal{Q}^{\Sigma}$ over $T$ can lift to $W$ locally on $T$. For this, we may assume that the underlying scheme of $T$ is the spectrum of a strict localization of a scheme of finite type over $\mathbb{Z}$. Further, by the Artin approximation theorem, we may assume that $T$ is the spectrum of a complete local ring $R$ with a separably closed residue field. Then, $W(R) \rightarrow$ $\mathcal{Q}^{\Sigma}(R)$ is isomorphic to $p^{\prime}: \lim W\left(R / m_{R}^{n}\right) \rightarrow \lim _{\longleftarrow} \mathcal{Q}^{\Sigma}\left(R / m_{R}^{n}\right)=\mathcal{Q}^{\Sigma}\left(R / m_{R}\right)$. Since $W$ is log smooth over $S$, each transition morphism $W\left(R / m_{R}^{n+1}\right) \rightarrow$ $W\left(R / m_{R}^{n}\right)$ is surjective by [4, Corollary 3.11]. Hence, $p^{\prime}$ is surjective, and $W \rightarrow \mathcal{Q}^{\Sigma}$ is also surjective.

\subsection{Partial group law}

Next, since $(W \times W)^{\prime}$ is represented by a log blowup of $W \times{ }_{S} W$, the morphism $(W \times W)^{\prime} \rightarrow W$ comes from some $i$. For each $x \in W$, write $\bar{x}$ for its image in $\mathcal{Q}$. Since the set of triples $(x, y, z) \in W \times W \times W$ such that the products $\bar{x} \bar{y}, \bar{x} \bar{y} \bar{z}$, and $\bar{y} \bar{z}$ in $\mathcal{Q}$ belong to $\mathcal{Q}^{\Sigma}$ is also represented by a proper fs log scheme, the morphism $(W \times W)^{\prime} \rightarrow W$ satisfies conditions (a)(c) in Section 4.3 over some $S_{i}$; that is, we may assume that the morphism $(W \times W)^{\prime} \rightarrow W$ is a partial group law. We may assume that this partial group law is compatible with that of $G$. Further, since $\mathcal{Q}^{\Sigma}$ is locally of finite presentation, we may assume that it is also compatible with the partial group law of $\mathcal{Q}^{\Sigma}$.

\subsection{The condition (f)}

Further, we consider the morphism $G \times{ }_{S_{i}} W \rightarrow W \times{ }_{\mathcal{Q}^{\Sigma}} W$ over $S_{i}$ in condition (f) in Section 4.3. This is an isomorphism over $S$, and $W \times{ }_{\mathcal{Q}^{\Sigma}} W$ over $S_{i}$ is represented by an open subscheme of a log blowup of $W \times{ }_{S_{i}} W$. Hence, this morphism is already an isomorphism over some $S_{i}$; that is, we may assume that $W$ is a $G$-torsor. Thus, we have an object of $\mathcal{B}$ in Proposition 4.4 over $S_{i}$.

\subsection{End of the proof of finiteness}

Now, by Proposition 4.4, this object corresponds to a log elliptic curve over $S_{i}$, which induces the original $E$ over $S$. 
Finally, we prove that $\mu$ comes from a level structure over some $S_{i}$. This is because the kernel of $n: E \rightarrow E$ is represented by $\lim _{\longleftarrow}\left(\{0\} \rightarrow E^{\left(\Sigma^{\prime}\right)} \stackrel{n}{\leftarrow} E^{\left(\Sigma^{\prime}\right)}\right)$, where $\Sigma^{\prime}$ is the collection $\{\{i / n\} \mid i \in \mathbb{Z}\}$.

\subsection{Proof of uniqueness}

We next prove statement (2). Let $\left(E_{i}, \mu_{i}\right) \in F(R)(i=1,2)$. Assume that for each $n$, the pullbacks of $\left(E_{i}, \mu_{i}\right)(i=1,2)$ to $R / m_{R}^{n}$ coincide. We have to prove that then $\left(E_{i}, \mu_{i}\right)$ coincide. We may assume that there is an exact sequence $0 \rightarrow G_{i} \rightarrow E_{i} \stackrel{f_{i}}{\rightarrow} \mathbb{G}_{m, \log }^{\left(q_{i}\right)} / \mathbb{G}_{m} q_{i}^{\mathbb{Z}} \rightarrow 0(i=1,2)$ on $R$ as in Definition 1.3(2). Then, $q:=q_{1}=q_{2}$ because $q_{1} \equiv q_{2} \bmod m_{R}^{n}$ for all $n$. Take $\Sigma=\{[(2 k-1) / 4,(2 k+1) / 4] \mid k \in \mathbb{Z}\}$, and let $W_{i}=E_{i}^{(\Sigma)}(i=1,2)$. We have $\left.\left.W_{1}\right|_{R / m_{R}^{n}} \cong W_{2}\right|_{R / m_{R}^{n}}$. We claim that $W_{1} \cong W_{2}$. We can see that $\left(M / \mathcal{O}^{\times}\right)_{\left.W_{1}\right|_{R / m_{R}^{n}}}$ is generated by global sections. To see this, we may assume that $n=0$ and that, in this case, $E_{i}^{(\Sigma)}$ is a base change of the universal object over $\mathbb{Z}\left[\zeta_{n}\right]\left[\left[q^{1 / n}\right]\right]$. For such a universal object, $M / \mathcal{O}^{\times}$is generated by global sections because it is a regular scheme endowed with the log structure by a simple normal crossing divisor. Hence, $\left(M / \mathcal{O}^{\times}\right)_{\left.W_{1}\right|_{R / m_{R}^{n}}}$ is generated by global sections, too. Then, $W_{1} \cong W_{2}$ by GAGF (see [7, Theorem 4.2.7]). Further, $f_{1}=f_{2}$ because $f_{1} \equiv f_{2} \bmod m_{R}^{n}$ for all $n$. Thus, $G_{1} \cong G_{2}$. The partial group laws on $W_{i}$ also coincide, and hence, by Proposition 4.4, we conclude that $A_{1} \cong A_{2}$. Level structures clearly coincide, too.

\section{§6. Drinfeld level structures, $\Gamma_{0}(N)$, and moduli stacks}

Here we sketch some variants. Our emphasis still lies in the fact that the concept of log elliptic curve makes degenerate stories parallel to the corresponding nondegenerate stories.

\section{1 .}

First, we briefly discuss Drinfeld $\Gamma_{*}(N)$-structures of log elliptic curves. Let $N \geq 1$.

Definition 6.1.1. Let $E$ be a log elliptic curve over an fs log scheme $S$.

(1) A $\Gamma(N)$-structure of $E$ is a triple $(D, P, Q)$, where

(a) $D$ is a subgroup sheaf of $E$ represented by a strict, finite locally free group scheme of rank $N^{2}$ over $S$; and

(b) $P, Q$ are two sections of order $N$ and generate $D$, that is, give a $(\mathbb{Z} / N \mathbb{Z})^{2}$-generator of $D$ in the sense of $[5$, Section 1.10.5]. (We regard $D$ as a group scheme without $\log$ structure.) 
(2) A $\Gamma_{1}(N)$-structure of $E$ is a pair $(C, P)$ of a finite locally free subgroup scheme $C$ and a section $P$ which is of order $N$ and generates $C$.

(3) $\mathrm{A} \Gamma_{0}(N)$-structure of $E$ is a finite locally free subgroup scheme $C$ which étale locally is generated by a section of order $N$.

REMARK 6.1.2.

(1) Over Spec $\mathbb{Z}[1 / N]$, the above Drinfeld $\Gamma_{*}(N)$-structures coincide with the ones in Definition $1.5(*=\emptyset, 1,0)$. They also coincide with those of Katz and Mazur [5] away from the cusps.

(2) It can be shown that for a log elliptic curve $E$, the subgroup scheme $\operatorname{Ker}(E \stackrel{N \text {-times }}{\longrightarrow} E)$ is, két locally, a finite flat group scheme of rank $N^{2}$. So $E$ has, két locally, $\Gamma_{*}(N)$ structures for any $N$ and $*=\emptyset, 1,0$.

(3) A natural question is whether a log elliptic curve with Drinfeld $\Gamma_{*}(N)$ structure in our sense corresponds to a generalized elliptic curve with Drinfeld $\Gamma_{*}(N)$-structure in Conrad's sense (see [1]).

6.1.3. Assume that $N \geq 3$ (resp., $N \geq 4$ ). As in Definition 2.1, we can define the moduli functor of $\log$ elliptic curves with Drinfeld $\Gamma_{*}(N)$ structures, where $*=\emptyset$ (resp., 1), and we think that these moduli functors are represented by fs log schemes which are proper and log smooth over Spec $\mathbb{Z}$. One can ask if this moduli space of ours coincides with the Conrad compactification over Spec $\mathbb{Z}$ with log structure defined by cusps.

\section{2 .}

Next, the case of $\Gamma_{0}(N)$ also can be treated in a way similar to the nondegenerate case. Let $M>2$ be an auxiliary integer which is prime to $N$. Then, $X_{0}(N) \times_{X(1)} X(M)$ is the fine moduli of the appropriate moduli problem over Spec $\mathbb{Z}[1 / N]$, formulated by using the $\Gamma_{0}(N)$-structure in Definition $1.5(3)$.

Using the Drinfeld level structure in Definition 6.1.1(3), we can work even over $\mathbb{Z}$.

We plan to study, in a forthcoming paper in this series, the higherdimensional analogue of $X_{0}(N) \times_{X(1)} X(M)$ (see Stroh [8]).

\section{3.}

Log stacks also can be considered in a way parallel to the nondegenerate case. Then, in a way similar to the nondegenerate case, we can construct moduli stacks of log elliptic curves with $\Gamma_{*}(N)$-structures for any $N \geq 1$. The auxiliary numbers $M$ as in Section 6.2 are not necessary for $\Gamma_{0}(N)$ (see Conrad [1]). 


\section{Appendix. Alternative construction of universal log elliptic curves}

In this appendix, we give an alternative construction of universal log elliptic curves over $X(N)$ over $\mathbb{Z}[1 / N]$ in Section 3 . Fix $N \geq 3$.

Let $\mathcal{E}$ be a universal generalized elliptic curve over $X(N)$ as in Section 2.2.

Below, roughly, we get our universal log elliptic curve as a quotient of the disjoint union of several variants of $\mathcal{E}$ by an equivalence relation. Contrary to the construction in Section 3, this alternative one is global (see Section A.1). In particular, it explains a precise relationship between our construction and Deligne and Rapoport's $\mathcal{E}$ (see [2]).

\section{A.1 Feature of the construction}

Recall that the construction in Section 3 is locally regarded as a quotient under a suitable equivalence relation of models $\mathcal{E}_{q^{i}}^{(1 / N) \mathbb{Z})}$ for larger and larger $i$. (We need only $\mathcal{E}_{q^{i}}^{(I)}$ with $I=(1 / N) \mathbb{Z}$, but in $[3$, Section 1$]$ we used all $I$ to simplify the description of the equivalence relation.) The alternative construction here is also regarded as a suitable quotient of models $\mathcal{E}_{q^{i}}^{((1 / N) \mathbb{Z})}$, but globally. We use the Deligne-Rapoport family $\mathcal{E}$ to have such models globally.

Before we explain the alternative construction, we introduce some notation and prove a property of $\mathcal{E}$.

Let us consider the sheaf $\mathcal{C}:=\mathbb{G}_{m, \log } / \mathbb{G}_{m}$ on $X(N)$ as in Section 4.1. For a bounded rational interval $I:=\left[\alpha, \alpha^{\prime}\right]$ of $\mathbb{Q}, \alpha, \alpha^{\prime} \in \mathbb{Q}$, we define a sheaf $\mathcal{C}^{I}$ as the subsheaf of $\mathcal{C}$ consisting of sections $x$ satisfying $q^{\alpha}|x| q^{\alpha^{\prime}}$. Further, we define $\overline{\mathcal{C}}_{1}:=\left(\bigcup_{l \in \mathbb{Z}} \mathcal{C}^{[l / N,(l+1) / N]}\right) / q^{\mathbb{Z}} \subset \mathcal{C} / q^{\mathbb{Z}}$. In the notation of [3, Section 1$]$, $\left.\overline{\mathcal{C}}_{1}\right|_{O_{v}}$ coincides with $\mathbb{G}_{m, \log }^{\left(q_{v},(1 / N) \mathbb{Z}\right)} /\left(\mathbb{G}_{m} q_{v}^{\mathbb{Z}}\right)$.

Lemma A.2. The sheaf $\mathcal{E} / \mathcal{G}$ is isomorphic to $\overline{\mathcal{C}}_{1}$. (Recall that $\mathcal{G}$ is the universal semi-abelian scheme over $X(N)$ as in Section 3.)

Proof. First note that $\overline{\mathcal{C}}_{1}$ is locally of finite presentation (see Section 5.6). Let $i$ be the inclusion map from the set $J$ of cusps to $X(N)$. Since $i^{-1} \mathcal{E}$ is isomorphic to the subsheaf of $\mathbb{G}_{m, \log } / q^{\mathbb{Z}}$ consisting of sections $x$ locally satisfying $q^{l / N}|x| q^{(l+1) / N}$ for some $l \in \mathbb{Z}$, and since $i^{-1} \mathcal{G}$ is sent to $\mathbb{G}_{m}$ by this isomorphism, the quotient $i^{-1}(\mathcal{E} / \mathcal{G})$ is isomorphic to $i^{-1} \overline{\mathcal{C}}_{1}$. Since $\overline{\mathcal{C}}_{1}=i_{*} i^{-1} \overline{\mathcal{C}}_{1}$, we have a homomorphism $h: \mathcal{E} / \mathcal{G} \rightarrow \overline{\mathcal{C}}_{1}$. We prove the bijectivity of this $h$. Let $U$ be an fs log scheme over $X(N)$, and we have to prove the bijectivity of $h(U)$. For this, first we may assume that the underlying scheme of $U$ is the spectrum of the strict Henselization $R$ of a local ring of a scheme of finite 
type over $\mathbb{Z}$. Next, by the Artin approximation theorem, we may assume further that $R$ is complete. Furthermore, we may replace $R$ with $R / m_{R}^{n}$ $(n \geq 1)$, where $m_{R}$ is the maximal ideal of $R$. In fact, assume that $h\left(R / m_{R}^{n}\right)$ is bijective for any $n \geq 1$. If $a, b \in \mathcal{E}(R)$ have the same image in $\overline{\mathcal{C}}_{1}(R)$, then, for each $n$, we have $a_{n}=b_{n} g_{n}$ in $\mathcal{E}\left(R / m_{R}^{n}\right)$ for some $g_{n} \in G\left(R / m_{R}^{n}\right)$, where $a_{n}$ and $b_{n}$ are the images of $a$ and $b$, respectively. Hence, $a=b g$ in $\mathcal{E}(R)$ if we put $g:=\left(g_{n}\right)_{n} \in \underset{\longleftarrow}{\lim } G\left(R / m_{R}^{n}\right)=G(R)$. Therefore, $h(R)$ is injective. On the other hand, since $\mathcal{E}$ is log smooth over $X(N)$, each transition homomorphism $\mathcal{E}\left(R / m_{R}^{n+1}\right) \rightarrow \mathcal{E}\left(R / m_{R}^{n}\right)$ is surjective by [4, Corollary 3.11]. Hence, $\mathcal{E}(R)=\lim _{\longleftarrow} \mathcal{E}\left(R / m_{R}^{n}\right) \rightarrow \mathcal{E}\left(R / m_{R}\right) \rightarrow \overline{\mathcal{C}}_{1}\left(R / m_{R}\right)$ is surjective. From this, we also see that $h(R)$ is surjective. Thus, it is enough to show that $h\left(R / m_{R}^{n}\right)$ is bijective. When the image of $\operatorname{Spec}\left(R / m_{R}^{n}\right)$ is not contained in $J$, this is trivial because $\mathcal{E}=\mathcal{G}$ there. Otherwise, $h\left(R / m_{R}^{n}\right)$ is also bijective because $i^{-1}(\mathcal{E} / \mathcal{G})=i^{-1} \overline{\mathcal{C}}_{1}$.

\section{A.3 Idea of the construction}

We explain the idea of the alternative construction. As we said above, the idea is that there should be a surjection from the disjoint union of the global versions of $\mathcal{E}_{q^{m}}^{((1 / N) \mathbb{Z})}$ (i.e., the global version of $\mathbb{G}_{m, \log }^{\left(q^{m},(1 / N) \mathbb{Z}\right)} / q^{m \mathbb{Z}}$ ), which we denote by $\mathcal{P}_{m}$, to the desired log elliptic curve. Once $\mathcal{P}_{m}$ is defined, the description of the equivalence is routine. To obtain $\mathcal{P}_{m}$, we want to divide $\mathbb{G}_{m, \log }^{(q,(1 / N) \mathbb{Z})} / q^{\mathbb{Z}}$ by $\mathbb{Z} / m \mathbb{Z}(1)$ to obtain $\mathbb{G}_{m, \log }^{\left(q^{m},(1 / N) \mathbb{Z}\right)} / q^{m \mathbb{Z}}$ via the multiplication-by- $m$ map, or we want to divide $\mathcal{E}_{q}^{((1 / N) \mathbb{Z})}$ by $\mathbb{Z} / m \mathbb{Z}(1)$ to obtain $\mathcal{E}_{q^{m}}^{((1 / N) \mathbb{Z})}$. That is, we want to divide $\mathcal{E}$ by $\mathbb{Z} / m \mathbb{Z}(1)$ to obtain $\mathcal{P}_{m}$. But an obstacle lies in the fact that $\mathcal{E}$ does not necessarily contain $\mathbb{Z} / m \mathbb{Z}(1)$. To overcome this difficulty, we work étale locally as follows.

\section{A.4 Fs log scheme $\mathcal{P}_{m}$}

We define $\mathcal{P}_{m}$. For a positive integer $m$ prime to $N$, consider the moduli functor $\mathfrak{X}(m, N)$ classifying injective homomorphisms $b_{m}: \mathbb{Z} / m \mathbb{Z}(1)_{U} \rightarrow$ $\mathcal{G} \times{ }_{X(N)} U$ for fs $\log$ schemes $U$ over $X(N)$. Then, $\mathfrak{X}(m, N)$ is represented by an fs $\log$ scheme over $X(N)$, denoted by $X(m, N)$, which is strict étale over $X(N)$. We denote by $b_{m}: \mathbb{Z} / m \mathbb{Z}(1) \rightarrow \mathcal{G} \times_{X(N)} X(m, N)$ the universal object over $X(m, N)$.

Let $P_{m}$ be the quotient scheme of $\mathcal{E}_{m}:=\mathcal{E} \times_{X(N)} X(m, N)$ over $X(m, N)$ by the action of the image of $b_{m}: \mathbb{Z} / m \mathbb{Z}(1) \rightarrow \mathcal{G} \times_{X(N)} X(m, N)$ endowed with the natural log structure. 
Let us denote by $\mathcal{P}_{m}$ the unique étale sheaf over $X(N)$ whose restriction to $X(m, N)$ is represented by $P_{m}$.

Then, over $Y(N)$, the pullback of $\mathcal{P}_{m}$ is $\mathcal{E} /(\mathbb{Z} / m \mathbb{Z}(1))$. Over $O_{v}$, the pullback of $\mathcal{P}_{m}$ is $\mathcal{E}_{q_{v}^{m}}^{((1 / N) \mathbb{Z})}$. Over $v$, the pullback of $\mathcal{P}_{m}$ is $\mathbb{G}_{m, \log }^{\left(q_{v}^{m},(1 / N) \mathbb{Z}\right)} / q_{v}^{m \mathbb{Z}}$. There is a natural map $\mathcal{P}_{m} \rightarrow \mathcal{C} / q^{m \mathbb{Z}}$ which is compatible with $\mathcal{E} \rightarrow \mathcal{C} / q^{\mathbb{Z}} \stackrel{m}{\rightarrow}$ $\mathcal{C} / q^{m \mathbb{Z}}$ (see Lemma A.2).

\section{A.5 Equivalence relation}

We define a $\log$ elliptic curve over $X(N)$, denoted by $\mathcal{E}_{2}^{\log }$ here, as a quotient of $\coprod_{(m, N)=1, m>0} \mathcal{P}_{m}$ by an equivalence relation $\sim$.

To describe the equivalence relation $\sim$, we introduce further notation.

Let $m$ be a positive integer prime to $N$. Let $I$ be a bounded rational interval of $\mathbb{Q}$ that is contained in $[l m / N,(l+1) m / N]$ for some integer $l$. We define $\mathcal{P}_{m}^{I}$ as the Cartesian product $\mathcal{P}_{m} \times_{\mathcal{C} / q^{m \mathbb{Z}}} \mathcal{C}^{I}$. Then, if $m^{\prime}=$ $m m^{\prime \prime}$, there is a natural map $\mathcal{P}_{m^{\prime}}^{I} \rightarrow \mathcal{P}_{m}$ whose restriction to $v$ is compatible with the canonical map $\mathbb{G}_{m, \log }^{\left(q_{v}^{m^{\prime}},(1 / N) \mathbb{Z}\right)} / q_{v}^{m^{\prime} \mathbb{Z}} \subset \mathbb{G}_{m, \log } / q_{v}^{m^{\prime} \mathbb{Z}} \rightarrow \mathbb{G}_{m, \log } / q_{v}^{m \mathbb{Z}}$ and whose restriction to $Y(N)$ is the multiplication-by- $m^{\prime \prime}$ map $\mathcal{E} /(\mathbb{Z} /$ $\left.m^{\prime} \mathbb{Z}(1)\right) \rightarrow \mathcal{E} /(\mathbb{Z} / m \mathbb{Z}(1))$.

Further, let $i$ be an integer. If $i+I$ is contained in some interval $\left[l^{\prime} m / N\right.$, $\left.\left(l^{\prime}+1\right) m / N\right]$ with an integer $l^{\prime}$, then the $q^{i}$-times morphism $\mathcal{P}_{m}^{I} \rightarrow \mathcal{P}_{m}$; $x \mapsto q^{i} x$ is defined.

\section{A.6 Alternative construction}

Now the alternative construction of the universal log elliptic curve $\mathcal{E}^{\log } \rightarrow$ $X(N)$ is $\mathcal{E}_{2}^{\log }:=\coprod_{(m, N)=1, m>0} \mathcal{P}_{m} / \sim$, where $\sim$ is the following equivalence relation: for an fs log scheme $U$ over $X(N)$, two $U$-sections $f_{1} \in \mathcal{P}_{m_{1}}$ and $f_{2} \in \mathcal{P}_{m_{2}}$ are equivalent if, étale locally on $U$, there exist a multiple $m_{3}$ of $m_{1}$ and of $m_{2}$, a $U$-section $f_{3}$ of $\mathcal{P}_{m_{3}}$, bounded rational intervals $I_{1}, I_{2}$ of $\mathbb{Q}$, and integers $i_{1}, i_{2}$, such that, for $k=1,2, I_{k}$ is contained in the interval $\left[l_{k} m_{k} / N,\left(l_{k}+1\right) m_{k} / N\right]$ for some integer $l_{k}, i_{k}+I_{k}$ is contained in the interval $\left[m_{3} / N,(l+1) m_{3} / N\right]$ for some integer $l$, the section $f_{k}$ can be lifted to a $U$-section $\tilde{f}_{k}$ of $\mathcal{P}_{m_{3}}^{I_{k}}$, and the $q^{i_{k}}$-times morphism $\mathcal{P}_{m_{3}}^{I_{k}} \rightarrow \mathcal{P}_{m_{3}}$ sends $\tilde{f}_{k}$ to $f_{3}$.

Proposition A.7. The above $\mathcal{E}_{2}^{\log }$ is canonically isomorphic to the universal log elliptic curve constructed in Section 3.

Proof. We denote by $\mathcal{E}_{1}^{\log }$ the universal log elliptic curve given in Section 3 . We prove that it is isomorphic to the above $\mathcal{E}_{2}^{\log }$. For this, we first 
construct a homomorphism $f: \mathcal{E}_{2}^{\log } \rightarrow \mathcal{E}_{1}^{\log }$. For each integer $m$ prime to $N$, by the definition of $\mathcal{E}_{m}$, we have a morphism $\left.\mathcal{E}_{m}\right|_{Y(N)} \rightarrow \mathcal{G}$, which induces an isomorphism $\left.\mathcal{P}_{m}\right|_{Y(N)}$ to $\mathcal{G}$. On the other hand, let $v \in J$. We have a morphism $\left.\mathcal{E}_{m}\right|_{O_{v}} \rightarrow \mathcal{E}_{q_{v}}$. It is easy to see that this morphism is equivariant with respect to the action of the image of $b_{m}$ in Section A.4 that acts trivially on the target and induces a morphism $\left.\mathcal{P}_{m}\right|_{O_{v}} \cong \mathcal{E}_{q_{v}^{m}}^{((1 / N) \mathbb{Z})} \rightarrow \mathcal{E}_{q_{v}}$.

So, using the diagram in Section 3.3, we have morphisms $\mathcal{P}_{m} \rightarrow \mathcal{E}_{1}^{\text {log }}$ and $\coprod_{(m, N)=1} \mathcal{P}_{m} \rightarrow \mathcal{E}_{1}^{\log }$.

We show that the last map kills the equivalence and factors through $\mathcal{E}_{2}^{\log }$. Let $f_{1} \in \mathcal{P}_{m_{1}}$ be equivalent to $f_{2} \in \mathcal{P}_{m_{2}}$. Assume that we are given the data $m_{3}, f_{3}$, and so forth, in the definition of the equivalence in Section A.6. Then, first, considering the diagram of natural maps

$$
\mathcal{P}_{m_{k}} \leftarrow \mathcal{E}_{q_{v}^{m_{3}}}^{\left(m_{k} /\left(N m_{3}\right) \mathbb{Z}\right)} \rightarrow \mathcal{P}_{m_{3}} \leftarrow \mathcal{E}_{q_{v}^{m_{3}}}^{\left((1 / N) \mathbb{Z} \cup\left(\alpha_{k} / m_{3}+\mathbb{Z}\right) \cup\left(\alpha_{k^{\prime}} / m_{3}+\mathbb{Z}\right)\right)} \leftarrow \mathcal{P}_{m_{3}}^{I_{k}}
$$

where $\left[\alpha_{k}, \alpha_{k}^{\prime}\right]:=I_{k}$, and applying equivalence relation (1) in Section 3.2, we see that the image of $f_{k}$ in $\mathcal{E}_{1}^{\log }$ and the image of $\tilde{f}_{k}$ in $\mathcal{E}_{1}^{\log }$ coincide. Further, by equivalence relation (2) in Section 3.2, the image of $\tilde{f}_{k}$ coincides with that of $f_{3}$. Thus, the images of $f_{k}$ in $\mathcal{E}_{1}^{\log }$ coincide, and we have the desired morphism $f: \mathcal{E}_{2}^{\log } \rightarrow \mathcal{E}_{1}^{\log }$.

We now prove that $f$ is an isomorphism. Taking the quotients of $\mathcal{E}_{2}^{\log }, \mathcal{E}_{1}^{\log }$ by $\mathcal{G}$, we get an exact sequence of sheaves

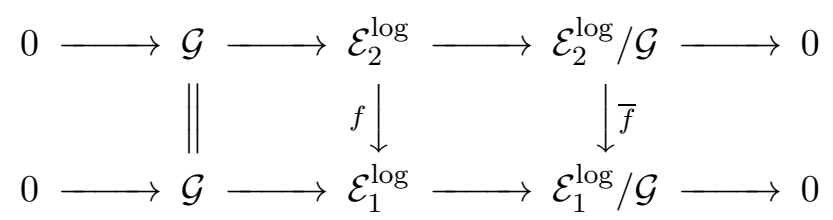

So, it is enough to show that $\bar{f}$ is an isomorphism. Fix $v \in J$. In the following, we work around $v$ and use the same symbols for the objects after pulling back to $\operatorname{Spec} O_{v}$. First, note that we already know that $\mathcal{E}_{1}^{\log } / \mathcal{G}$ is $\mathbb{G}_{m, \log }^{\left(q_{v}\right)} /\left(\mathbb{G}_{m} q_{v}^{\mathbb{Z}}\right)$. Since the image of $\mathcal{P}_{m}$ in this quotient is $\mathbb{G}_{m, \log }^{\left(q_{v}^{m},(1 / N) \mathbb{Z}\right)} /\left(\mathbb{G}_{m} q_{v}^{\mathbb{Z}}\right)$, the surjectivity of $\bar{f}$ follows. To see the injectivity, it is enough to show that $\mathcal{E}_{2}^{\log } / \mathcal{G} \rightarrow \mathcal{C} / q^{\mathbb{Z}}$ is injective. Let $f_{k} \in \mathcal{P}_{m_{k}}(k=1,2)$, and assume that their images coincide in $\mathcal{C} / q^{\mathbb{Z}}$. It suffices to prove that their images coincide in $\mathcal{E}_{2}^{\log } / \mathcal{G}$. Let $m_{3}$ be a multiple of $m_{1} m_{2}$. For $k=1,2$, take an interval $I_{k}=\left[\alpha_{k}, \alpha_{k}^{\prime}\right]$ of the form $\left[l_{k} m_{k} / N,\left(l_{k}+1\right) m_{k} / N\right]$ for some integer $l_{k} \geq 0$ 
such that $f_{k}$ can be lifted to some $\tilde{f}_{k} \in \mathcal{P}_{m_{3}}^{I_{k}}$. Let $g_{k}$ be the image of $\tilde{f}_{k}$ in $\mathcal{P}_{m_{3}} / \mathcal{G} \subset \mathcal{C} / q^{m_{3} \mathbb{Z}}$. By the assumption, we may assume that $g_{1}=q^{i} g_{2}$ for some $i \geq 0$. Replace $m_{3}$ by its multiple such that $i+I_{2}$ is contained in $\left[0, m_{3} / N\right]$. Let $f_{3}$ be the image of $\tilde{f}_{1}$ in $\mathcal{P}_{3}$. By adjusting $f_{2}$ by the action of $\mathcal{G}$, we may assume further that the image of the $q^{i}$-times morphism sends $\tilde{f}_{2}$ to $f_{3}$. Hence, $f_{1}$ and $f_{2}$ are equivalent by definition, which implies the desired injectivity of $\mathcal{E}_{2}^{\log } / \mathcal{G} \rightarrow \mathcal{C} / q^{\mathbb{Z}}$.

Acknowledgments. We appreciate the careful reading and the many useful comments from the referees.

\section{REFERENCES}

[1] B. Conrad, Arithmetic moduli of generalized elliptic curves, J. Inst. Math. Jussieu 6 (2007), 209-278. MR 2311664. DOI 10.1017/S1474748006000089.

[2] P. Deligne and M. Rapoport, "Les schémas de modules de courbes elliptiques" in Modular Functions of One Variable, II (Antwerp, 1972), Lecture Notes in Math. 349, Springer, Berlin, 1973, 143-316. MR 0337993.

[3] T. Kajiwara, K. Kato, and C. Nakayama, Logarithmic abelian varieties, Nagoya Math. J. 189 (2008), 63-138. MR 2396584.

[4] K. Kato, "Logarithmic structures of Fontaine-Illusie" in Algebraic Analysis, Geometry, and Number Theory (Baltimore, 1988), Johns Hopkins University Press, Baltimore, 1989, 191-224. MR 1463703.

[5] N. M. Katz and B. Mazur, Arithmetic Moduli of Elliptic Curves, Ann. of Math. Stud. 108, Princeton University Press, Princeton, 1985. MR 0772569.

[6] C. Nakayama, Logarithmic étale cohomology, Math. Ann. 308 (1997), 365-404. MR 1457738. DOI 10.1007/s002080050081.

[7] M. C. Olsson, Log algebraic stacks and moduli of log schemes, Ph.D. dissertation, University of California, Berkeley, Berkeley, California, 2001. MR 2702292.

[8] B. Stroh, Compactification minimale et mauvaise réduction, Ann. Inst. Fourier (Grenoble) 60 (2010), 1035-1055. MR 2680823.

Takeshi Kajiwara

Department of Applied Mathematics

Faculty of Engineering

Yokohama National University

Yokohama 240-8501

Japan

kajiwara@ynu.ac.jp 
Kazuya Kato

Department of Mathematics

University of Chicago

Chicago, Illinois, 60637

USA

kkato@math.uchicago.edu

Chikara Nakayama

Department of Mathematics

Tokyo Institute of Technology

Tokyo 152-8551

Japan

cnakayam@math.titech.ac.jp 